\title{
Perspectives on Interpersonal Care Among People Who Obtain Abortions Through Clinical and Accompaniment Models in Argentina
}

Chiara Bercu ( $\boldsymbol{D}$ cbercu@ibisreproductivehealth.org )

Ibis Reproductive Health https://orcid.org/0000-0001-8329-8954

\section{Sofia Fillipa}

Ibis Reproductive Health

Ana Maria Ramirez

Ibis Reproductive Health

Anna Katz

University of California Berkeley School of Law

Colectiva Feminista La Revuelta (Belén Grosso Ruth Zurbriggen)

Colectiva Feminista La Revuelta

Sandra Vázquez

Grupo FUSA

\section{Sarah E. Baum}

Ibis Reproductive Health

\section{Research}

Keywords: abortion, accompaniment, reproductive health, person-centred care, quality of care, Argentina

Posted Date: April 13th, 2021

DOl: https://doi.org/10.21203/rs.3.rs-391415/v1

License: (c) (i) This work is licensed under a Creative Commons Attribution 4.0 International License. Read Full License 


\section{Abstract}

\section{Introduction:}

Little is known about how people who obtain abortion services perceive quality and what aspects of interpersonal care matter to them. This qualitative study aimed to understand preferences among people seeking abortions at a reproductive health clinic and an accompaniment model of self-managed abortion in Argentina.

\section{Study Design:}

We conducted 24 in-depth interviews with people who obtained abortion care in Neuquén and Buenos Aires, Argentina. We iteratively coded transcripts using a thematic analysis approach based on interpersonal domains present in current quality of care frameworks.

\section{Results}

Participants described high-quality abortion care as feeling acompañamiento and contención from their providers - terms that imply receiving kind, caring, compassionate and emotionally supportive care throughout their abortions. They described four key elements of interpersonal interactions: attentive communication from providers and accompaniers, clear and understandable information provision, nonjudgmental support, and individualized options for pain management.

\section{Conclusions}

People obtaining abortions in two distinct models of care in Argentina consistently identified receiving compassionate and supportive care throughout an abortion as a key aspect of care. The findings have implications for incorporating client perspectives in the development of care guidelines, training of providers, and monitoring and improving of services. This is particularly important as the government of Argentina prepares to expand legal access to abortion.

\section{Plain English Summary}

Little is known about how people who obtain abortion services perceive the quality of their care and what aspects of interpersonal interactions with providers matter to them. This qualitative study aimed to understand preferences among people seeking abortions at a reproductive health clinic and an accompaniment model of self-managed abortion in Argentina. We interviewed 24 people who obtained abortion care in Neuquén and Buenos Aires, Argentina. We asked people about their experiences of abortion care and their preferences for care. Then we analyzed the interviews, specifically assessing aspects of interpersonal care based on quality of care frameworks from the literature. 
Participants described high-quality abortion care as feeling acompañamiento and contención from their providers - terms that imply receiving kind, caring, compassionate and emotionally supportive care throughout their abortions. They described four key elements of interpersonal interactions: attentive communication from providers and accompaniers, clear and understandable information provision, nonjudgmental support, and individualized options for pain management.

In conclusion, people obtaining abortions in two distinct models of care in Argentina consistently identified receiving compassionate and supportive care throughout an abortion as a key aspect of care. The findings have implications for incorporating client perspectives in the development of care guidelines, training of providers, and monitoring and improving of services. This is particularly important as the government of Argentina prepares to expand legal access to abortion.

\section{Background}

In Argentina, abortion was legally restricted and regulated by the Penal Code up until January 2021. Prior to the change in law, a legal abortion was only available if the pregnancy put the pregnant person's life and/or health at risk, or if the pregnancy was a product of rape. While some provinces in the country permitted the legal termination of pregnancy when the person's physical, emotional, or social wellbeing would be harmed by carrying the pregnancy to term, many provinces did not. Abortion services are provided through a range of models in Argentina including comprehensive reproductive health clinics and hospitals which provide legal abortion services based on the legal exceptions, and accompaniment groups which offer in-person and virtual information and support for people across the country who were self-managing medication abortions ${ }^{1,2}$. Despite the legal restrictions, abortion in Argentina remained common and people had safe and effective abortions in both models of care. However, little is known about how people perceive the quality of their abortion services and what matters to them in their interactions with providers.

Existing research on abortion quality of care has highlighted the importance of interpersonal care; interpersonal care refers to the client's experiences and perceptions of the healthcare interaction ${ }^{3}$. A systematic review of published indicators for measuring quality of abortion services found the measures largely focused on structural and process aspects, such as infrastructure and technical competence, and only one third of the indicators were developed by incorporating abortion clients' preferences. Indicators to measure interpersonal care have not been consistently included; as such, there are gaps in our understanding of what aspects of the client provider interaction matter most to clients ${ }^{4,5}$. The Interpersonal Quality in Abortion Care scale was developed in 2019 and sought to fill this gap by creating a measure derived from patients' perspectives in the United States; the scale includes items related to respect, feeling listened to, and kindness ${ }^{4}$. These domains highlight the importance of interpersonal interactions in person-centered care.

Person-centered care is defined as an approach to care that is oriented around the patient's identified needs, expectations and preferences ${ }^{6}$. At the root of person-centered care lie the interpersonal interactions 
between people and their providers, and providers' ability to center the patient and their needs during care. Studies addressing person-centered care in contraceptive and maternal health services have shown that women value interpersonal factors such as trust, empathy, respect, support, confidentiality and nonjudgmental care from providers ${ }^{7-10}$ and that these shape perceptions of quality and future care seeking behavior $^{11-13}$. There is more recent evidence emerging within the context of abortion demonstrating how client experiences of quality are positively impacted by person-centeredness ${ }^{14}$, such as receiving comprehensive counseling, non-judgmental care and supportive care, as well as feelings of autonomy and choice ${ }^{15-17}$. Though limited in number, studies from Latin America focusing on patient perspectives of abortion quality have found that client-provider interactions and information provision were identified as some of the most salient aspects of patients' experiences ${ }^{18,19}$. One study on quality of post-abortion care in public hospitals in Argentina found that, generally, care was not provided in a respectful manner and that key aspects, such as information provision, were not present. ${ }^{19}$

Despite what we know about the importance of interpersonal care in quality of care, there are gaps in understanding preferences and priorities among people seeking abortion in different contexts and models of care, particularly outside of the healthcare systems. Given the lack of recent data in Argentina, we sought to analyze interviews with people who had abortion care in both comprehensive reproductive health clinics and through accompaniment groups. This analysis is particularly timely given the change in the law permitting abortion up to 14 weeks; we hope these qualitative data can inform integration of person-centered care in the implementation of legal abortion care throughout the country. This study aimed to gain a deeper understanding of how people obtaining abortion experienced interactions with providers and what aspects of interpersonal care they valued.

\section{li. Methods}

In this study, we analyzed semi-structured in-depth interviews with people who obtained an abortion in Buenos Aires and Neuquén, Argentina. These interviews were part of a larger study conducted in four countries: Argentina, Bangladesh, Ethiopia and Nigeria. In the large study, we conducted a total of 98 interviews in order to gain a deeper understanding of women's experiences with abortion services and their perceptions of quality of care. The interview guide was developed based on quality of care frameworks $5,20,21$ as well as previous studies on abortion quality. The guide was similar across the four contexts, but edits were made for clarity or cultural applicability in partnership with each local research team.

Participants in Argentina were recruited from two different abortion care organizations - through a feminist accompaniment group based in Neuquén and a comprehensive reproductive health clinic in Buenos Aires. The feminist accompaniment group (also referred to as an abortion hotline), Colectiva Feminista, La Revuelta (La Revuelta), hosts group information sessions where women learn how to have a safe self-managed abortion and complete a medication abortion (MA) at home. Trained accompaniers provide support and information by telephone throughout the medication abortion process to individuals 
seeking an abortion. In this paper, the term "accompaniers" is used to refer to members of the feminist collective who provided abortion information and support through La Revuelta. The reproductive health clinic, Casa FUSA, Centro para Atención de Adolescentes y Jóvenes (FUSA), provides in-clinic abortion care in Buenos Aires. Although FUSA also offers medication abortion, for this investigation we only recruited individuals who had received a manual vacuum aspiration (MVA), a common procedure for induced abortion or post-abortion care ${ }^{22}$. Women who had their abortions with FUSA generally visited the clinic prior to their abortions to participate in a group information session, then had an individual interview with a provider and returned a few days later for their MVA. In this paper, the term "providers" is used to refer to both medical personnel and staff who provide abortion care at FUSA.

We conducted 24 semi-structured in-depth interviews, 12 at each recruitment site, from November 2018 to January 2019. Trained staff at each recruitment site recruited participants prospectively, during group information sessions, or retrospectively, after they had completed their abortions. People were eligible for the study if they were 15 years or older, able to provide informed consent, able to speak Spanish, and had had an abortion within 6 months of recruitment. One interviewer (BG) who was trained in qualitative methods conducted all of the interviews in person at a space provided by the recruitment partner or in a secure location of the participant's choosing. BG, was associated with La Revuelta at the time of the study, but did not provide counseling to any of the people recruited to participate. The interviews lasted between 40 and 60 minutes and were audio-recorded. Participants were compensated with a publictransportation card with a value of approximately \$10 USD. This study was approved by Allendale Investigational Review Board based in the United States and Comité de Bioética de la Fundación Huésped, based in Argentina.

We conducted thematic analysis in order to explore client perceptions and experiences of interpersonal care during their abortions. All interviews were professionally transcribed and analyzed thematically in Spanish. We developed an initial codebook using key themes from the interview guide, domains of quality of care drawn from a literature review on the subject, and emergent codes from the transcripts. The codebook was developed for the larger four-country study and applied across all the interviews. For the data in Argentina, two researchers (CB and SF) coded the same two transcripts, after which they met to discuss discrepancies in coding, adjusted, redefined, or modified codes or code descriptions and then applied the final codebook to all 24 transcripts. Coding was conducted with MAXQDA 2018 (VERBI Software, 2019). Themes from across the applied codes were analyzed during a process of writing code summaries and assessing patterns as well as outlying trends within the data set. Key staff at recruitment sites contributed to the interpretation of data by reviewing preliminary findings and discussing themes. Quotes have been translated into English. Original quotes, in Spanish, are available in Appendix A.

\section{lii. Results}

Amongst the 24 participants, the average age was 30 years old, ranging between 20 and 41 years old. $\mathrm{A}$ quarter of participants reported having an abortion after 12 weeks' gestation. Half of all participants had a medication abortion (all recruited from the accompaniment model) and half had a Manual Vacuum 
Aspiration (MVA) (all recruited from the clinic). There were six participants who reported having a prior abortion and nine who already had children. None of the participants reported being married, however nearly half of participants reported being in a relationship, four of whom lived with their partners (Table 1).

Table 1

Participant characteristics

\begin{tabular}{|c|c|}
\hline & $\begin{array}{l}N=24 \\
n(\%)\end{array}$ \\
\hline \multicolumn{2}{|l|}{ Age (years) } \\
\hline Mean & 30 \\
\hline Range & $20-41$ \\
\hline \multicolumn{2}{|l|}{ Abortion method } \\
\hline Medication abortion & $12(50.0 \%)$ \\
\hline Manual vacuum aspiration (MVA) & $12(50.0 \%)$ \\
\hline \multicolumn{2}{|l|}{ Gestational age } \\
\hline$<=12$ weeks & $16(66.6 \%)$ \\
\hline$>12$ weeks & $6(25.0 \%)$ \\
\hline No data & $2(8.3 \%)$ \\
\hline \multicolumn{2}{|l|}{ Previous abortion } \\
\hline Yes & $6(25.0 \%)$ \\
\hline No & $18(75.0 \%)$ \\
\hline \multicolumn{2}{|l|}{ Children } \\
\hline Yes & $9(37.5 \%)$ \\
\hline No & $15(62.5 \%)$ \\
\hline \multicolumn{2}{|l|}{ Marital Status } \\
\hline Married & $0(0.0 \%)$ \\
\hline In a relationship & $11(45.8 \%)$ \\
\hline Single & $13(54.2 \%)$ \\
\hline
\end{tabular}

\section{Acompañamiento and Contención[1]}

Universally, participants recruited from both the clinic and accompaniment models of care described high-quality abortion care as feeling acompañamiento and contención from their providers. These terms 
emerged over and over in the descriptions of positive interactions, and were highlighted across the sample as necessary for good care.. Interpersonal care provided with acompañamiento and contención helped participants trust their provider or accompanier, which, in turn, reassured their sense of safety throughout the abortion. When describing her experience with the hotline, a participant explained, "I always say that they provide you with a security that, out there, perhaps at another place, I do not know if...let's see, they are not doctors, but the fact that they provide such contención is really important, you do not feel alone." (Age 34, Accompaniment). Another participant, who did not experience any abortion symptoms after three medication abortion attempts, echoed the importance of knowing that they would not be left alone. With the support of their accompanier, the participant ultimately ended up receiving an MVA at a clinic.

"What I always felt was that sense of security, you are not going to come out of this alone, we are never going to abandon you, this is where we solve this. That kind of...gives you that sense of security, to say I failed once and now what do I do, maybe they will leave me on my own, no, they will not leave you alone, because they told me, 'we already started to dance, we are going to continue dancing until the song is over', and that is how it is." (Age 33, Accompaniment)

Acompañamiento and contención also played a role in relieving participants' anxieties. Participants described a sense of respite that was rooted in the realization that they would be able to end their pregnancies in a context of high-quality care. A participant described, "[...] I felt...how can I say it, very well attended to, I did not expect to get here, very emotionally contenida..." (Age 26, Clinic). Finally, a participant expressed that they felt "light" after meeting with the accompaniment group, since they knew that the accompaniers would help them terminate their pregnancy,

"I felt... I felt light, I felt supported, acompañada, and I knew that everything would be fine, so I felt calmer, much calmer, because I was very scared, I felt that my problem was solved, that is what I felt." (Age 26, Accompaniment).

Below we present four key aspects of abortion care with acompañamiento and contención which help to define what participants in Argentina valued during interpersonal interactions: attentive communication from providers and accompaniers, clear and understandable information provision, non-judgmental support, and individualized options for pain management.

\section{Attentive communication and interactions}

Many participants in the study described feeling supported during interpersonal interactions when providers and accompaniers communicated continuously and were attentive to their needs throughout their abortion process, particularly during the abortion procedure itself. One participant at the clinic appreciated the fact that the provider explained every step of their MVA as it was taking place, and showed attentiveness to their comfort, 
"Well, I can't remember the name of the girl who was accompanying me, but well, she was sitting next to me, talking to me, as if we knew each other forever, the doctor too, who was there, was telling me everything she was doing. That everything was going well. Always asking me if it hurt, what I felt, if I felt something strange."(Age 25, Clinic)

When providers and accompaniers showed concern about their physical and emotional wellbeing, participants felt safer, calmer and more trusting of the provider or accompanier. One participant highlighted the fact that their provider asked for permission to touch them before beginning the MVA procedure, which they did not anticipate. This helped them feel that they were receiving respectful care; they explain, "[...] they were very attentive because they told me, 'I'm going to put this on you, I'm going to put that on you, I'll touch you here.' So these are things that you are not accustomed to happening either. No specialist is going to ask you for your permission" (Age 41, Clinic).

Participants accompanied by the feminist accompaniment group described feeling contención and acompañamiento while interacting with their accompanier from the beginning to the end of their experience. This participant explained the importance of knowing the accompanier would be there for them if something happened,

"From the day you are going to do it, they are already accompanying you, that is, it is not that they are just going to show you how to do it [...] there is a before, during and after, and everything is with

acompañamiento, and that is really good, because you know that in the moment if something happens to you it is not that you go to a hospital or something, you have someone to consult, who can help you." (Age 30, Accompaniment)

In some cases, participants were surprised by how supported they felt by the interactions they had with accompaniers over phone calls and text, such as this participant,

"[...] but it was like... it was very relieving and I felt the contención beyond the kilometers and beyond the non-contact face to face, I felt the contención and I knew they were there, it was not the only time they called me, [...], they explained everything to me, they called me before, after the process, and during the process too [...]" (Age 30, Accompaniment)

The attentiveness that both accompaniers and providers showed towards participants helped them feel that they could rely on the providers or accompanier, which helped participants feel that they were very well cared for and supported during their abortions.

\section{Comprehensive, clear information provision}

Almost all the participants described the importance of receiving clear and comprehensive information from their providers or accompaniers. At both recruitment sites, comprehensive information, which included details on each step of the abortion process, was provided during an initial group information session, as this participant explains, 
"They explained everything step by step, and it was all like, 'yes, let's go, I want to end [my pregnancy] here', and nothing, in terms of contención here I found it $100 \%$, that is, contención that I did not find in him [my partner], that I did not find in my friend, in the psychologist." (Age 20, Clinic)

For the participant, the detailed information they received on the procedure helped them make the choice to have their abortion at the clinic. Additionally, this participant linked information provision to feeling contención at the clinic, highlighting how providing transparency on the abortion procedure may have facilitated a sense of trust between the participants and the providers.

Participants also appreciated the opportunity to ask questions and discuss their circumstances with providers and accompaniers, as well as with other people who were there to have an abortion. One participant mentioned that they were given the opportunity to interact with the MVA instruments prior to the procedure in the clinical model. Additionally, the group workshops that both models used, offered a space for participants to hear what questions other people had and instilled in them a sense of community and safety. As one participant explained,

"This thing of it being in a group makes you feel a little more relaxed, because you know that you are not alone and that there others are going to ask questions that do not occur to you and that could happen to you, so, then you are like more sure of what you are doing [...]" (Age 30, Accompaniment).

Various participants also highlighted the importance of receiving information that was easily accessible and understandable, as this participant explains, "It was like they spoke to you in a way that you could understand everything, you understand? And I came without knowing anything...the truth is that they inspired so much confidence in me, so much security, they explained everything, absolutely everything[...]" (Age 32, Clinic). Receiving thorough information helped participants to feel safe, helped them trust their providers and helped them feel well-prepared for their abortion experiences.

\section{Empathetic and non-judgmental listening}

Participants often mentioned the importance of feeling heard by their providers or accompaniers. This made them feel respected and understood by their providers and accompaniers. One participant explains, "[...] they provide contención emotionally, they listen to you, they know how to listen, they know how to understand the situation you are going through and professionally they are very delicate, respectful, attentive [...]" (Age 26, Clinic). Another participant described how it felt to be able to share their motives for wanting an abortion with a provider who helped them feel that their experience was important,

"[...] I know that a lot of girls pass through here, but they like made you feel, I don't know, not special, but like it mattered what you were feeling, and that your decision was fine, whatever it was, that the reason why you didn't want to have it [the pregnancy] mattered [...]" (Age 20, Clinic)

Perceiving that providers and accompaniers did not cast judgement or stigmatize their decision, and instead listened empathetically, helped participants feel that they were receiving care with acompañamiento and contención. 
Participants also recounted that in both models of care, they felt that providers and accompaniers took efforts to normalize abortion. One participant explains of the accompaniment model, "[...] since here [abortion] is normalized, what we do is not judged in any way" (Age 33, Accompaniment). The group workshops were also instrumental in normalizing abortion. One participant who was initially skeptical about the group workshop recounts how they later understood it as a way of legitimizing abortion,

"'Why do I have to go to a collective interview?' At first I didn't like that one bit and after, I understood why it was collective, or at least I explained it to myself through the experience we had, and I liked that...it's like half because it's good thinking from the perspective of taking it [abortion] out of the dark or the clandestine or illegal practice or secret practice, the fact that it is collective [...]" (Age 36, Clinic)

Another participant accompanied by the hotline model further explained how the group workshops helped to create a friendly rapport between participants and accompaniers, "[...] the girls made it more enjoyable, it seemed like a chat with friends. Yes, it was really good. Yes, I felt very good, I felt contenida, it was good" (Age 30, Accompaniment). The non-judgmental space that providers and accompaniers helped to facilitate during group workshop, as well as the empathetic listening that participants recounted receiving from their providers and accompaniers, helped them feel heard, validated and supported and was an important aspect of receiving care with acompañamiento and contención.

\section{Choice in pain management}

Participants reported feeling that providers and accompaniers took their needs into account and offered innovative techniques for pain management during their abortions. For instance, participants who received care at the clinic were given a number of options for pain management including hot water bottles to place on their abdomens and the opportunity to play the music of their choice during their MVAs. One participant described how the music and the conversation with the people in the room helped distract them from the pain,

"They put music on for me, Los Redondos [a band], and the truth is that if I'm honest with you, I didn't feel any pain, [...] the girls talked to me and we chatted, and I had the hot water bottle, for like in case you feel pain." (Age 32, Clinic)

When reflecting in the interviews, participants often highlighted these moments as representative of supportive interpersonal interactions during their abortion care. Another participant who received care at the clinic described being surprised at how much effort her providers made to meet her individual comfort needs,

"They told me something that really surprised me, they said 'if you want, we can play music', which surprised me in the best sense...at what level they are, I don't know how to say it, as if they are really thinking about the comfort of the patient, in a moment like that, I had the best time possible..." (Age 21, Clinic) 
As this participant points out, the ability for many participants to have choice in their pain management helped them feel as though they had the best possible healthcare experience. In the feminist accompaniment group, accompaniers suggested that participants try different pain management options at home, for example using a hot compress, or setting up the space in which they were going to take the medications so that it would feel unique and comfortable. For instance, one participant mentioned watching their favorite TV show and preparing some of their favorite snacks, as their accompanier had suggested,

"She [the accompanier] told me to stay calm, that the calmer I was, the faster and easier it would be, she told me to create a nice ambiance, that the person who is with you transmits good things to you, if you like incense, light an incense stick, prepare whatever you like to eat [...] I made myself a cake of dulce de leche [caramel], walnuts and chocolate...she told me watch whatever you want, so I did it watching Ru Paul...[television show]" (Age 30, Accompaniment)

The empathic and compassionate way through which providers and accompaniers centered the needs and choices of participants in relation to pain management helped them feel as though their individual needs were being taken into account and helped them feel that they were in a safe and comfortable environment during their abortions.

\section{Footnote:}

[1] These are two key terms in Spanish that participants used frequently to describe the way that providers/accompaniers interacted with them. In an effort to capture the essence of these words, we will continue to refer to the two terms in Spanish throughout this paper. "Contención", and its adjective "contenida" literally translate to "containment" in English. However, in use, the word suggests a type of kind, caring, compassionate emotional support, a support that contains the individuals feeling of fear or anxiety. "Acompañamiento", and its adjective "acompañada" literally translate to "accompaniment" in English. But in practice, it too suggests kind, caring, and supportive care that accompanies one throughout their healthcare experience.

\section{Iv. Discussion}

The results of this qualitative study provide insights into the elements of interpersonal care that were most salient for people having abortions through clinical and accompaniment models of care in Argentina. The specific examples of positive interactions with providers and accompaniers help shed light on what clients look for in a good abortion experience. Participant's highlighted open communication, comprehensive information provision, non-judgmental care and choice in pain management as key components of their abortion experiences. Study participants consistently reiterated how imperative it was and how much they appreciated interpersonal care that was provided with acompañamiento and contención - continual emotional support, empathy, and understanding. 
These findings contribute to a growing body of literature that recognizes interpersonal care as a priority and documents the value of person-centered, empathetic, and emotionally supportive interactions with providers. Our results echo a recent study with abortion clients in Kenya that highlighted the importance of receiving respectful, supportive care continuously throughout their abortion process ${ }^{16}$. The findings encourage us to consider quality of care, and specifically interpersonal care, as necessary not only during a single encounter with the provider, but from the time a person decides to have an abortion until the process is complete. This might include various types of providers for an individual, such as conversations with a primary care physician or hotline counselors, front desk staff, ultrasound technician, pharmacists selling abortion pills, or providers offering follow-up services. Our findings complement other studies that show that clients appreciate compassionate and supportive communication that is provided not just in-person but also virtually by telephone, online platforms or texting systems ${ }^{2324}$. In legally restrictive settings where people seeking abortion may not have access to legal clinical services, and particularly during restrictions associated with the global pandemic, these alternative mechanisms for communication will likely become more relevant and therefore quality must be monitored.

This study highlights various innovative ways of providing person-centered interpersonal interactions. Participants from both the clinical model and the accompaniment model noted the unique approach of providing group information sessions prior to an abortion. While some people were hesitant at first, nearly all participants felt the sessions were beneficial; they listened to each other's questions, felt less isolated, and the group atmosphere may have contributed to normalizing abortion experiences amongst participants. In addition, some participants reported being impressed by the providers' and accompaniers' attempts to make the abortion experience not only comfortable, but even enjoyable. This included playing music during in-clinic abortions, offering hot water bottles during and after their procedures, and providing suggestions for how to set up their spaces at home to ease the self-managed abortion experience. These innovative approaches centered client's needs and helped them feel cared for and supported during their abortions. Strategies that help to normalize abortion and offer both choice and autonomy to people seeking abortions are particularly noteworthy within abortion care as they help people feel empowered in their decisions and may help tackle some of the internalized stigma or social stigma surrounding abortion.

This study adds nuance to domains that have emerged in person-centered care frameworks in the literature. The details offered in these narratives have implications for how we develop or adapt tools to measure quality of care from the client's perspective. For example, in addition to receiving comprehensive information about the abortion process, participants felt that it was important that the information be provided in a way that helped them feel heard, comforted, validated, and free to ask clarifying questions. Participants also noted their appreciation for having choice in pain management during their abortion both at the clinic during surgical abortion and at home for medication abortions. If indicators simply address whether a person received information or pain management, they may not successfully assess how they received that element of care. Conversely, if indicators simply ask if a person felt they received respectful compassionate care, they may not successfully capture at what point during the process the 
person did, or did not, feel respected. Sudhinaraset et al. recently developed and validated a personcentered abortion care scale based on data from abortion clients in Kenya that included sub-scales to measure respectful and supportive care as well as communication and autonomy ${ }^{25}$. These domains are reflected in our data, which suggests that key aspects of person-centered care may carry over across political and social contexts. However, there are some context-specific findings that may be relevant for adaptations of the scale. The detailed descriptions of high-quality care from this study in Argentina can contribute to future research exploring how this scale or future measures could be adapted for the Argentinian context. This is particularly important as the government prepares to expand legal abortion access in public facilities across the country. It will be critical to incorporate the values and preferences of people who obtain abortion in the development of care guidelines, training of providers, and monitoring and improving of services.

There are a number of limitations that should be considered when interpreting the findings in this study. These data present a range of people's experiences aborting with support from either a clinic or an accompaniment group, both of which are based in urban areas, therefore the perspectives may not be representative of other models of care or of rural areas of Argentina. In addition, individuals recruited from the clinical model of care were receiving services at a private facility as such, the study does not offer insight into people's experiences of abortion in the public health system. Lastly, providers and accompaniers at the two sites were responsible for inviting people to participate in the study, which may have influenced who decided to participate and could have limited the negative feedback shared in the interviews.

\section{Conclusion}

This study identified elements of interpersonal care that were most salient to people seeking abortions at clinics and with accompaniment groups in Argentina. Our findings suggest that receiving compassionate and supportive care throughout an abortion was consistently a key aspect of care. While cultural and legal contexts may shift what is most important for people seeking abortions, we posit that the feeling of trust, empathy and respect from providers, which emerged from this study, is applicable across settings and models of care. Given the global shift towards medication abortion and the recent expansion of telemedicine during the Covid-19 global pandemic, this study reminds us that interpersonal interactions are highly important for people's overall experience of quality of care even when abortion support is being provided virtually. Furthermore, given this critical moment in abortion provision in Argentina, we hope that these findings will inform the provision and evaluation of person-centered abortion care in the country.

\section{Vi List Of Abbreviations}

Casa FUSA, Centro para Atención de Adolescentes y Jóvenes (FUSA): A comprehensive reproductive health clinic in Buenos Aires

Ibis Reproductive Health (Ibis) 
Collectiva Feminista La Revuelta (La Revuelta)

Manual Vacuum Aspiration (MVA)

Medication Abortion (MA)

\section{Vii Declarations}

Ethics approval and consent to participate: This study was approved by Allendale Investigational Review Board based in the United States and Comité de Bioética de la Fundación Huésped, based in Argentina. All participants provided verbal consent to participate in the study.

Consent for publication: Not applicable.

Availability of data and materials: The dataset generated and analyzed during the current study is not publicly available in order to maintain confidentiality and reduce risk to the participants. The interview guide is available from the corresponding author on reasonable request.

Competing interests: The authors declare that they have no competing interests.

Funding: Support for this work came from the David and Lucile Packard Foundation and the Children's Investment Fund Foundation. The funders had no role in the design, writing or decision to publish.

Authors' contributions: CB \& SF contributed to study design, coding, analysis, and write up of the manuscript. AR \& AK contributed to study design and codebook development, and revised preliminary drafts of the manuscript. BG \& RZ contributed to study design, recruitment, data collection, interpretation of findings, and revised preliminary drafts of the manuscript. SV contributed to recruitment and interpretation of findings. SB contributed to the study design, coding, analysis and write up of the manuscript. All authors read and approved the final manuscript.

Acknowledgements: We would like to thank our colleague, María Victoria Simón for supporting with data recruitment at Casa FUSA. We would also like to thank all of the members of the Colectiva Feminista La Revuelta and of Casa FUSA who made this study possible. Finally, we thank the ASQ research partners, Ipas and Metrics for Management, for their contributions to study design and conceptualization.

\section{Viii References}

1. Gomperts R, Jelinska K, Davies S, Gemzell-Danielsson K, Kleiverda G. Using telemedicine for termination of pregnancy with mifepristone and misoprostol in settings where there is no access to safe services. BJOG An Int J Obstet Gynaecol. 2008;115(9):1171-1178. doi:10.1111/j.14710528.2008.01787.x

2. Dzuba IG, Winikoff B, Peña M. Medical abortion: A path to safe, high-quality abortion care in Latin America and the Caribbean. Eur J Contracept Reprod Heal Care. 2013;18(6):441-450. 
doi:10.3109/13625187.2013.824564

3. Darney BG, Kapp N, Andersen K, et al. Definitions, measurement and indicator selection for quality of care in abortion. Contraception. 2019;100(5):354-359. doi:10.1016/j.contraception.2019.07.006

4. Donnelly KZ, Dehlendorf C, Reed R, Agusti D, Thompson R. Adapting the Interpersonal Quality in Family Planning care scale to assess patient perspectives on abortion care. J Patient-Reported Outcomes. 2019;3(1):3. doi:10.1186/s41687-018-0089-7

5. Dennis A, Blanchard K, Bessenaar T. Identifying indicators for quality abortion care: A systematic literature review. J Fam Plan Reprod Heal Care. 2017;43(1):7-15. doi:10.1136/jfprhc-2015-101427

6. WHO | What are integrated people-centred health services? WHO. Published online 2018. Accessed February 22, 2021. http://www.who.int/servicedeliverysafety/areas/people-centred-care/ipchswhat/en/

7. Holt K, Zavala I, Quintero X, et al. Women's preferences for contraceptive counseling in Mexico: Results from a focus group study. Reprod Health. 2018;15(1). doi:10.1186/s12978-018-0569-5

8. Renfrew MJ, McFadden A, Bastos $\mathrm{MH}$, et al. Midwifery and quality care: Findings from a new evidence-informed framework for maternal and newborn care. Lancet. 2014;384(9948):1129-1145. doi:10.1016/S0140-6736(14)60789-3

9. Jain AK, Hardee K. Revising the FP Quality of Care Framework in the Context of Rights-based Family Planning. Stud Fam Plann. 2018;49(2):171-179. doi:10.1111/sifp.12052

10. Holt K, Dehlendorf C, Langer A. Defining quality in contraceptive counseling to improve measurement of individuals' experiences and enable service delivery improvement. Contraception. 2017;96(3):133137. doi:10.1016/j.contraception.2017.06.005

11. Dehlendorf C, Henderson JT, Vittinghoff $\mathrm{E}$, et al. Association of the quality of interpersonal care during family planning counseling with contraceptive use. Am J Obstet Gynecol. 2016;215(1):78.e178.e9. doi:10.1016/j.ajog.2016.01.173

12. Abdel-Tawab N, Roter D. The relevance of client-centered communication to family planning settings in developing countries: Lessons from the Egyptian experience. Soc Sci Med. 2002;54(9):1357-1368. doi:10.1016/S0277-9536(01)00101-0

13. Holt K, Caglia JM, Peca E, Sherry JM, Langer A. A call for collaboration on respectful, person-centered health care in family planning and maternal health Prof. Suellen Miller. Reprod Health. 2017;14(1):13. doi:10.1186/s12978-017-0280-y

14. Sudhinaraset M, Landrian A, Montagu D, Mugwanga Z. Is there a difference in women's experiences of care with medication vs. manual vacuum aspiration abortions? Determinants of person-centered care for abortion services. Gemmill A, ed. PLoS One. 2019;14(11):e0225333. doi:10.1371/journal.pone.0225333

15. Taylor D, Postlethwaite D, Desai S, et al. Multiple Determinants of the Abortion Care Experience: From the Patient's Perspective. Am J Med Qual. 2013;28(6):510-518. doi:10.1177/1062860613484295

16. Cotter SY, Sudhinaraset M, Phillips B, et al. Person-centred care for abortion services in private facilities to improve women's experiences in Kenya. Cult Health Sex. 2021;23(2):224-239. 
doi:10.1080/13691058.2019.1701083

17. Regmi K, Madison J. Ensuring patient satisfaction with second-trimester abortion in resource-poor settings. Int J Gynecol Obstet. 2010;108(1):44-47. doi:10.1016/j.ijgo.2009.08.005

18. Becker D, Díaz-Olavarrieta C, Juárez C, García SG, Sanhueza P, Harper CC. Clients' perceptions of the quality of care in Mexico City's public-sector legal abortion program. Int Perspect Sex Reprod Health. 2011;37(4):191-201. doi:10.1363/3719111

19. Ponce de León RG, Billings DL, Barrionuevo K. Woman-centered post-abortion care in public hospitals in Tucumán, Argentina: Assessing quality of care and its link to human rights. Health Hum Rights. 2006;9(1):175-201. doi:10.2307/4065395

20. Kruk ME, Kelley E, Syed SB, Tarp F, Addison T, Akachi Y. Measuring quality of health-care services: What is known and where are the gaps? Bull World Health Organ. 2017;95(6):390-390A. doi:10.2471/BLT.17.195099

21. Sudhinaraset M, Afulani P, Diamond-Smith N, Bhattacharyya S, Donnay F, Montagu D. Advancing a conceptual model to improve maternal health quality: The person-centered care framework for reproductive health equity. Gates Open Res. 2017;1. doi:10.12688/gatesopenres.12756.1

22. Medical Management of Abortion.; 2018. Accessed January 22, 2021. http://apps.who.int/

23. Baum SE, Ramirez AM, Larrea S, et al. "It's not a seven-headed beast": abortion experience among women that received support from helplines for medication abortion in restrictive settings. Health Care Women Int. 2020;41(10):1128-1146. doi:10.1080/07399332.2020.1823981

24. Fix L, Seymour JW, Sandhu MV, Melville C, Mazza D, Thompson TA. At-home telemedicine for medical abortion in Australia: A qualitative study of patient experiences and recommendations. BMJ Sex Reprod Heal. 2020;46(3):172-176. doi:10.1136/bmjsrh-2020-200612

25. Sudhinaraset M, Landrian A, Afulani PA, Phillips B, Diamond-Smith N, Cotter S. Development and validation of a person-centered abortion scale: The experiences of care in private facilities in Kenya. BMC Womens Health. 2020;20(1). doi:10.1186/s12905-020-01071-w

\section{Supplementary Files}

This is a list of supplementary files associated with this preprint. Click to download.

- QuotesinSpanishandEnglishAppendixA.xlsx

- SRQRChecklist.docx 